\title{
Diagnosis of Intracranial Stenosis
}

\author{
Jay K. Joshi and Shyam Prabhakaran*
}

Department of Neurological Sciences, Rush University Medical Center, Chicago, IL 60612, USA

\begin{abstract}
The diagnosis of intracranial stenosis has improved with the advent and availability of non-invasive vascular imaging tests. Vascular imaging modalities to consider include transcranial Doppler, magnetic resonance and computed tomographic angiography. While these have strengths and weaknesses, they provide anatomic information. Physiologic and/or hemodynamic data can be gleaned from perfusion imaging, vasomotor reactivity testing, positron emission tomography, and quantitative magnetic resonance angiography. These latter tests may be particularly useful in stratifying stroke risk in individuals with intracranial stenosis. While most cases are due to atherosclerotic disease, the astute clinician still needs to consider other etiologies including vasoconstriction, vasculitis, and dissection before embarking on treatment decisions.
\end{abstract}

Keywords: Magnetic resonance angiography, computed tomographic angiography, stroke risk, hemodynamics.

\section{INTRODUCTION}

The diagnosis of intracranial stenosis has improved with the advent and availability of non-invasive vascular imaging tests. Conventional catheter-based angiography remains the gold standard but has potentially serious risks and limited availability. Hence, magnetic resonance angiography (MRA), computerized tomographic angiography (CTA), and transcranial Doppler (TCD) are the most commonly employed non-invasive modalities in the diagnostic evaluation of intracranial stenosis. Each has its own attributes and limitations. Besides their utility in the anatomic diagnosis of intracranial stenosis, non-invasive imaging can also provide critical physiologic information (such as blood flow characteristics) which can assist in prognostication and recurrent stroke risk stratification (Fig. 1). In this review, we will consider the role of non-invasive screening, discuss established and emerging cerebrovascular imaging tools for the diagnosis of intracranial stenosis, and determine their role in management.

\section{DIFFERENTIAL DIAGNOSIS}

Despite the ability to make the anatomic diagnosis of intracranial stenosis, one still needs to differentiate among several known radiographic mimics of atherosclerotic intracranial stenosis such as partial recanalization of an embolus and non-atherosclerotic diseases such as dissection, vasculitis, vasospasm, and fibromuscular dysplasia. For example, central nervous system vasculitis, whether primary or secondary, can result in multifocal and distal sites of arterial narrowing that results in a "beaded" appearance, while athero-stenosis typically involves the proximal circle of Willis vessels and vertebrobasilar circulation (i.e. middle cerebral, proximal or mid basilar, distal vertebral, and distal internal carotid arteries). Reversible cerebral vasoconstriction syndromes are a generalized, self-limiting

*Address correspondence to this author at the Department of Neurological Sciences, Rush University Medical Center, Chicago, IL 60612, USA; Tel: 312-942-4500; Fax: 312-563-2206; E-mail: shyam_prabhakaran@rush.edu group of disorders often due to vaso-active substances [1] that cause multifocal vasoconstrictive changes in the intracranial vasculature presenting with symptoms such as thunderclap headache and focal neurologic signs. Fibromuscular dysplasia and dissection, which typically affects the extracranial carotid arteries, is also a rare cause nonatherosclerotic stenosis in the intracranial circulation [2]. While a careful clinical history and physical examination can reasonably exclude many mimics, assuring high diagnostic certainty remains challenging. In some instances, repeat imaging may be required to evaluate mimic conditions that improve over time (i.e. complete recanalization of an embolus or resolution of vasospasm) versus persistent or worsening stenosis due to atherosclerosis.

Though atherosclerotic stenosis is the most common cause of intracranial arterial narrowing, assigning the appropriate stroke mechanism in a patient with intracranial atherosclerotic stenosis (i.e. determining whether the stenosis is symptomatic or asymptomatic) may still be far from straightforward. Besides large artery atherosclerosis, other stroke subtypes, particularly cardioembolic and lacunar strokes can co-exist in patients with intracranial stenosis. Furthermore, though recurrent strokes tend to have the same mechanism of action as the initial stroke event, up to $19 \%$ may be due to another mechanism including cardioembolism, extracranial large artery disease, and lacunar disease [3].

Consequently, cardioembolic and small-vessel etiologies need to be excluded in making the initial diagnosis of symptomatic intracranial stenosis and later in assessing the mechanism of recurrent ischemic events. These data suggest that even in patients with unequivocal intracranial atherosclerotic stenosis, the mechanisms of stroke can be heterogenous and assigning causative etiology with absolute certainty may be difficult.

\section{SCREENING}

Intracranial stenosis is responsible for $5-10 \%$ of all ischemic strokes in the United States and up to $50 \%$ among 
certain ethnic sub-types in Asia [4,5]. When symptomatic, it is associated with a $10-20 \%$ mortality rate [6-8]. Given the prevalence and severity of this disease, it is attractive to consider non-invasive neuroimaging as a means to screen atrisk populations before symptoms occur. For instance, diabetics, men, smokers, and non-Caucasians have the highest prevalence of intracranial stenosis [9-12] and may benefit from selected screening for the disease. However, the data supporting the benefits of asymptomatic screening are limited at best.

The risk of an ischemic stroke in asymptomatic intracranial stenosis is ten-fold less than that associated with symptomatic intracranial stenosis [13, 14]. However, in patients harboring both symptomatic and asymptomatic stenoses, $73 \%$ of recurrent ischemic strokes occurred in the symptomatic stenotic arterial territories while $27 \%$ were in previously asymptomatic regions [3]. Among out-of-territory recurrent strokes, $48 \%$ developed strokes distal to previously asymptomatic stenoses [3]. While the stroke risk due to asymptomatic intracranial stenosis is certainly lower than symptomatic stenosis, the actual risk is difficult to quantify and patients with asymptomatic stenosis coexisting with symptomatic stenosis may require careful risk assessment. Thus, despite the lack of robust, prospective data, selective screening for asymptomatic stenoses may be useful in highrisk populations (i.e. diabetics) but generalized screening of the population is likely not cost-effective or beneficial [11, 12]. Further study assessing long-term outcomes following screening for intracranial stenosis is warranted.

\section{ANATOMIC DIAGNOSIS OF INTRACRANIAL STENOSIS (FIG. 2)}

The gold standard of neurovascular imaging remains the conventional (catheter-based) angiogram, or digital subtraction angiography (DSA) [15]. In comparing the efficacy of non-invasive imaging techniques, DSA is considered the reference standard. It provides excellent overall visualization of vessel contour, anatomic localization, measurement of degree and length of stenosis, and assessment of collateral circulation. Modern DSA also incorporates three-dimensional reconstructed images from the raw data to provide even greater detail. However, due to its invasive nature, there is a small but real risk (approximately $0.7 \%$ ) of peri-procedural neurologic injury associated with DSA [16]. The most serious complications (ischemic and hemorrhagic stroke), should they occur, are noted within 24-72 hours of the procedure. In addition, DSA carries the nephrotoxic risk associated with iodinated

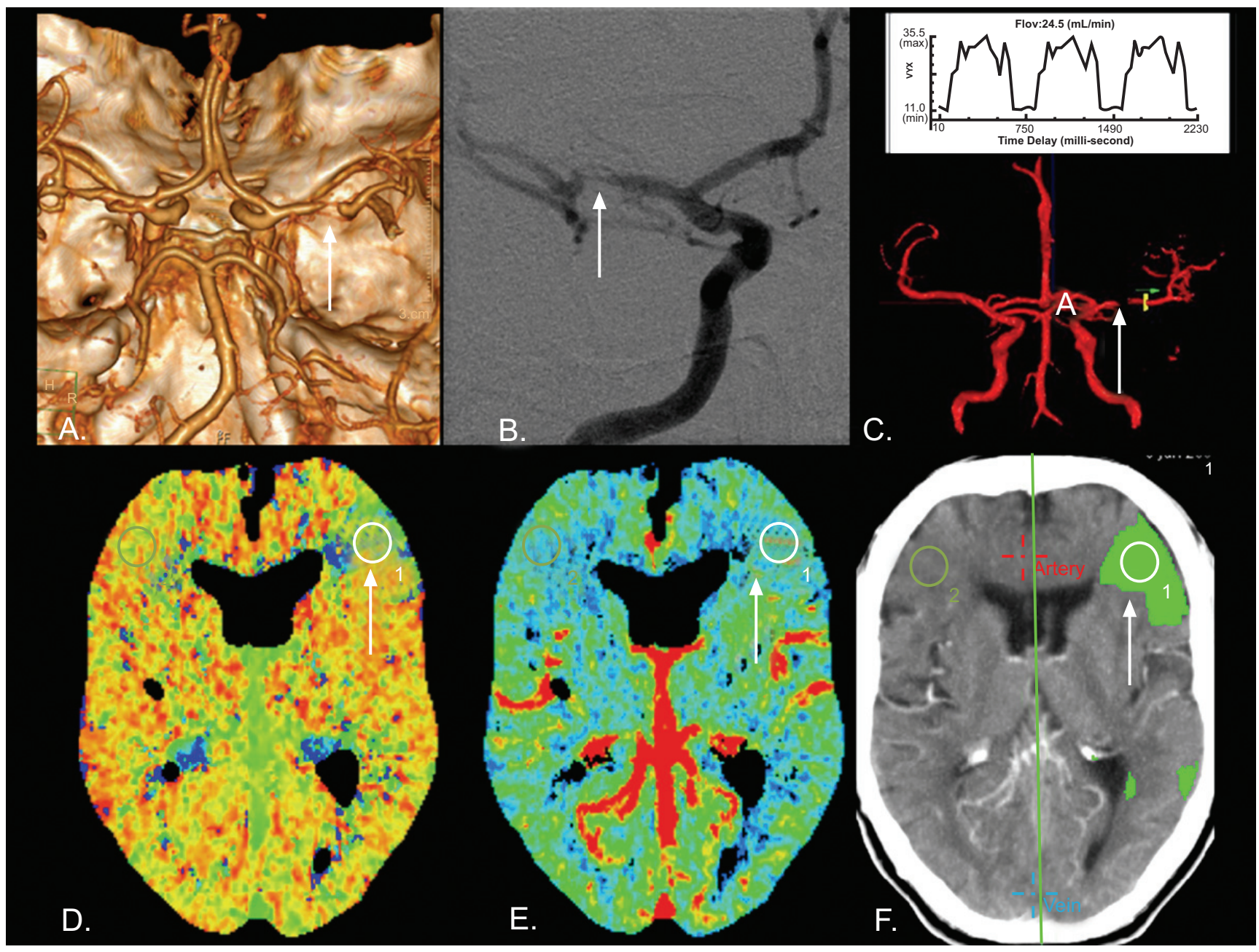

Fig. (1). Examples of imaging modalities (A, CTA; B, DSA; C, QMRA; D-F, CT perfusion). Arrows (white) indicate stenosis (A-C) and area of hypoperfusion (D-F). 
contrast injection and the potential for access-related injury (i.e. groin hematoma and limb ischemia). Given these concerns and the limited availability of qualified cerebral angiographers, the diagnosis of intracranial stenosis was previously inferred without anatomic visualization or neglected altogether until recently when non-invasive imaging overcame many of these concerns.

Transcranial Doppler is a widely available and inexpensive non-invasive test used to diagnose intracranial stenosis. It is based on the principle that flow velocity increases with worsening arterial stenosis (decreasing vessel diameter). It is commonly used to screen for cerebral vasospasm following subarachnoid hemorrhage but has demonstrated utility in sickle cell anemia, brain death, microembolic signal detection, enhanced clot lysis, as well as the diagnosis of intracranial stenosis [17, 18]. The location and degree of stenosis is inferred by the transducer probe's position, depth, direction, and from the measured velocity and contour of the waveform within an arterial segment. However, since direct anatomic visualization is not performed with TCD, its performance depends greatly on technical expertise and experience. In large controlled studies, the sensitivity, specificity, positive and negative predictive values of TCD are as follows: $73-92 \%, 89-99 \%$, $36-50 \%$, and $85-86 \%$, respectively $[17,19]$. For distinguishing between low and high grade stenosis, the sensitivity, specificity, and NPV of TCD are slightly lower: $82 \%, 73 \%, 79 \%$ respectively [20]. Given the high specificity but low positive predictive values, TCD can serve as a reasonable test to exclude intracranial stenosis but often requires another confirmatory test (often another noninvasive test or DSA) when the diagnosis of intracranial stenosis is suggested.

Magnetic resonance angiography is another commonly employed non-invasive imaging modality used to identify intracranial stenosis. The technique measures flow signal intensity as a function of proton spin, as utilized in time-offlight (TOF) MRA to visualize changes in the flow of blood. As a consequence, motion artifact, directional changes in arterial flow (i.e. from vertical to horizontal) can render the results ambiguous or even erroneous [15]. Its advantages are the absence of radiation exposure or radiocontrast risks [21]. Its major disadvantage, besides vulnerability to motion artifacts, is its inability to distinguish between high-grade stenosis and occlusion. A flow gap on TOF-MRA may represent high-grade stenosis or occlusion. In addition, it has limited to no use among patients who are morbidly obese, claustrophobic, or have implanted foreign metallic objects (i.e. pacemakers). Despite these limitations, MRA have shown $70 \%$ sensitivity in detecting intracranial stenosis > $50 \%$ and $81 \%$ sensitivity in detecting intracranial occlusion relative to DSA [15]. Overall, studies suggest MRA has the following performance characteristics in diagnosing intracranial stenosis: sensitivity $62-88 \%$, specificity $86-97 \%$, PPV 59-66\%, and NPV 87-88\% [15, 19, 22]. In clinical practice, MRA is considered a more useful modality than TCD, but given its low PPV, still requires further confirmatory diagnostic testing such as a second non-invasive test or DSA.

New innovations are expanding the role of MRA in intracranial stenosis. Contrast-enhanced MRA uses gadolinium dye to provide better anatomic visualization and overcome regions of changing blood flow directions [23]. Currently, it is the most widely used technique to visualize the external carotids [22] but it has been increasingly applied to the intracranial circulation. Quantitative MRA, utilizing phase-contrast techniques, has shown promise as a diagnostic test of intravascular blood flow to diagnosis hemodynamic failure in posterior circulation stenosis [24, $25]$ and screen for post-procedural stenosis at sites of stents or coils [26]. QMRA can also assess regional blood flow which can indicate the overall cerebral hemodynamic and collateral flow status [25]. Another emerging MR technique is high resonance imaging (HR-MRI), capable of visualizing and characterizing plaque morphology and behavior, and discriminating from other non-atherosclerotic etiologies [27, 28]. Further study using these novel modalities may increase the diagnostic utility of MRA and provide valuable prognostic data.

Compared to traditional time-of-flight MRA, CTA provides better anatomic visualization of the intracerebral vasculature $[15,29]$. While MRA focuses on flow motion dynamics, CTA focus on the relative penetrance of radiocontrast agents within the blood vessels and image the vessels in the form of a "vessel cast" [15]. While it can overcome the limitation of accurate visualization of slowflow lesions (i.e. high-grade stenosis), CTA can be degraded by local calcium or metallic artifacts [30]. Compared to DSA, the high sensitivity (98\%), specificity (99\%), PPV (93\%), and NPV (100\%) of CTA make this an excellent, and perhaps preferred, non-invasive diagnostic test for intracranial stenosis (Fig. 1) [29, 30]. A major disadvantage is the small risk of radiocontrast reactions and possible severe anaphylaxis [20]. In addition, nephrotoxicity is a known complication that can arise from contrast dye administration. Therefore, it may be contraindicated in those with chronic renal insufficiency, pregnancy, or known contrast allergies.

The relative availability, costs, advantages and disadvantages, along with the pre-test probability and test performance characteristics, will determine which test is best suited for an individual patient; however, other circumstances may also guide the choice of non-invasive imaging modality. For example, CTA can better visualize high-grade stenoses than time-of-flight MRA since the latter tends to overestimate the degree of stenosis and has lower sensitivity and specificity $[15,22,29,30]$. Furthermore, the relative accuracy of each test can vary depending on the location of the stenosis. The petrous ICA and cavernous ICA are susceptible to bony or calcium artifacts, and in this region, MRA tends to be more useful than CTA [15].

\section{PHYSIOLOGIC IMAGING MARKERS OF STROKE RISK}

As discussed earlier (Chapter 1), the mechanisms of ischemia in intracranial stenosis include progressive arterial narrowing, plaque instability (thrombo-embolism), and/or attenuated vasomotor reactivity due to impaired collateral flow. It is likely that these mechanisms interact in complex ways and co-exist in the same patient [31-33]. Identifying physiologic markers of the underlying vascular mechanism may be critical to determine stroke risk, develop mechanism- 


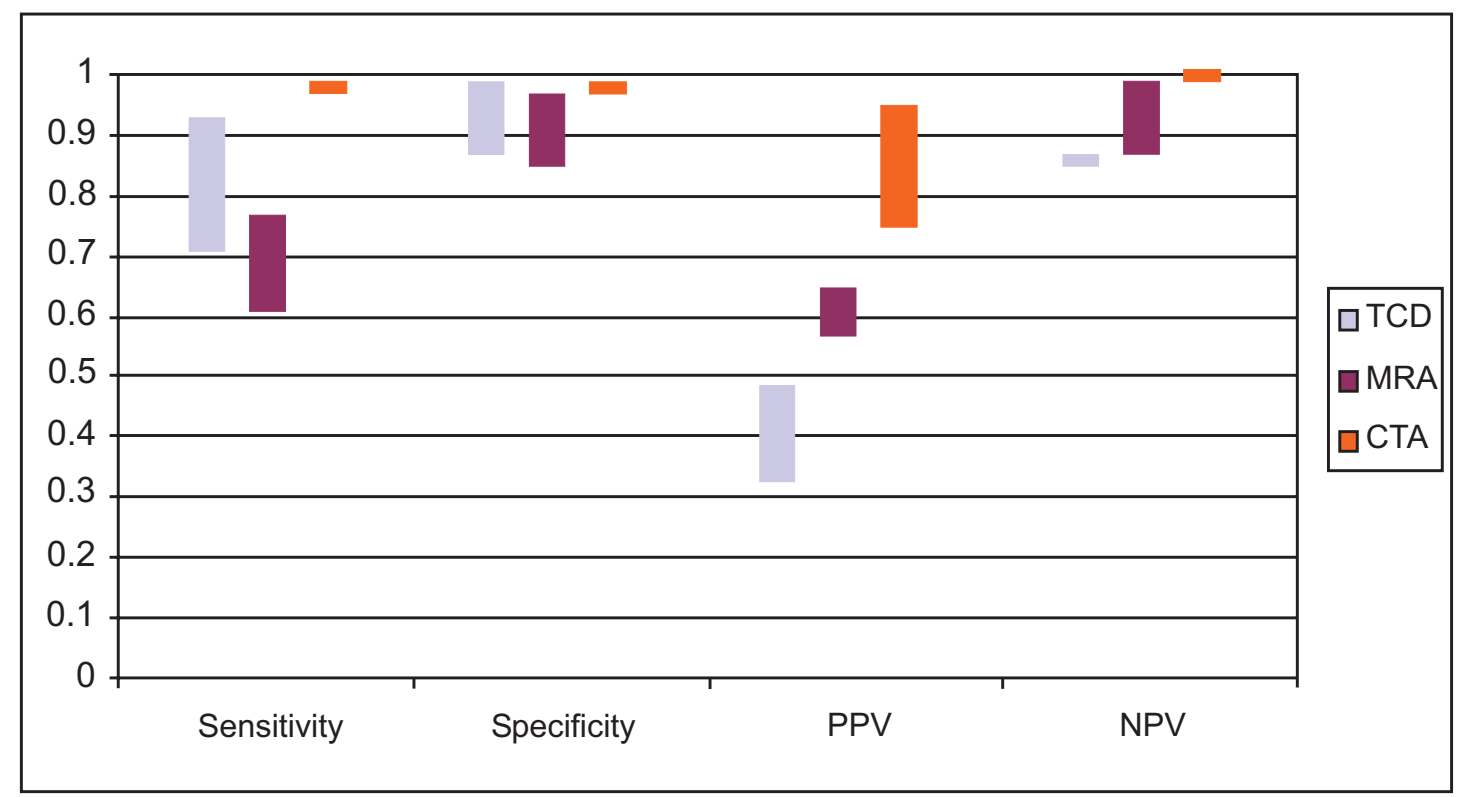

Fig. (2). Comparison of test performance for transcranial Doppler, magnetic resonance angiography, and computerized tomographic angiography in diagnosis of intracranial stenosis (conventional angiography is the reference standard); PPV-positive predictive value, NPVnegative predictive value.

specific prevention and treatment strategies, and assist in patient selection for endovascular therapies. Non-invasive imaging can provide critical physiologic data on anterograde flow, tissue perfusion, collateral flow, plaque morphology, and embolic signals and help in the determination of stroke mechanism (Fig. 3).

Physiologic information related to the viability of cerebral blood flow distal to a stenotic lesion factoring in collateral blood flow would also be an important variable to estimate the natural history risk for recurrent stroke. Two stages of hemodynamic failure have been described [34]. Stage 1 involves vasodilation in response to poor perfusion which results in increased cerebral blood volume and prolongation of mean transit time to preserve cerebral blood flow and oxygen extraction fraction. Stage 2 shows falling cerebral blood flow and increased oxygen extraction fraction. Early investigations with hemodynamic imaging suggested that compromised cerebral blood flow reactivity predicted an increased risk of stroke [35]. A recent small study compared interventional treatment of patients who failed medical therapy with those who demonstrated impaired regional cerebral blood flow (CBF) and found a non-significant trend toward better long-term outcome in those with impaired regional CBF [36].

Anterograde blood flow at and distal to the site of stenosis can be measured and quantified by QMRA or phasecontrast MRA. In the setting of arterial stenosis, intravascular blood flow decreases with advancing stenosis. QMRA can quantify the physiologic significance of the anatomic degree of stenosis [24, 26]. For example, a patient with $60 \%$ stenosis who has preserved anterograde flow is at a lower risk of ischemic stroke than a patient with $60 \%$ stenosis and diminished anterograde flow.

Phase-contrast MRA exploits the phase shift in the signal of flowing blood, which is proportional to flow velocity. Phase velocity maps can be constructed for quantitative measurements of volumetric flow rate (VFR) through medium and large-sized vessels. The technique of blood flow quantification by phase-contrast MRA is now commercially available through software called NonInvasive Optimal Vascular Analysis (NOVA, VasSol, Inc.,) and has been shown to be a reliable method for quantifying flow in the cerebral vasculature [37]. Volumetric blood flow measurement $(\mathrm{mL} / \mathrm{min})$ integrates flow velocity over the cross-sectional vessel area which correlates strongly with $\mathrm{CBF}$ at the tissue level.

Over the past decade, many studies have been performed using QMRA in the assessment of extracranial and intracranial arterial flow in patients with carotid stenosis and occlusion, vertebrobasilar ischemia, subclavian steal phenomenon, and following treatments with carotid endarterectomy (CEA), stenting, and bypass surgery [24, 25, 38-41]. Recent reports have also demonstrated the utility of QMRA in the measurement of pre- and post-procedure vessel flow following angioplasty for vertebrobasilar stenosis and Wingspan stent placement for extracranial vertebral artery stenosis [42, 43].

The largest of these studies assessed the risk of recurrent ischemic stroke in 47 patients with posterior circulation ischemic stroke or TIA and $>50 \%$ stenosis or occlusion of the vertebral or basilar arteries (72\% intracranial disease). The authors utilized a QMRA algorithm to stratify risk by VFR measured at baseline. Low flow ( $>20 \%$ decrease from normal values) correlated with increasing degree of stenosis: among 23 patients with $80-99 \%$ stenosis, 8 (34.8\%) had low flow while none of the 8 patients in the $50-80 \%$ stenosis group had low flow. At 24 months, 97.5\% of those with normal QMRA flow rates were event- free, compared to $81.5 \%$ with low flow. In Cox proportional hazards models, QMRA flow status predicted event-free survival independent of other covariates [24]. 


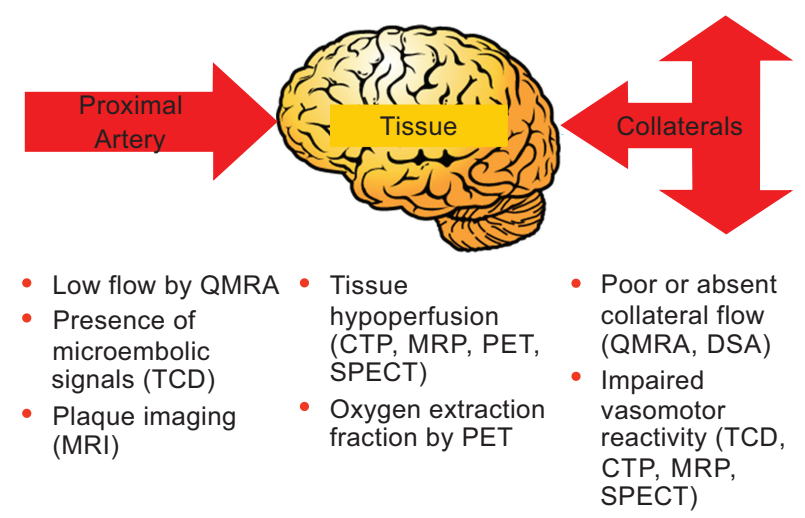

Fig. (3). Schematic of physiologic markers that may help stratify stroke risk among patients with intracranial stenosis.

Transcranial Doppler can also characterize anterograde blood flow by measuring mean flow velocity and detecting embolic signals (ES). Initially, velocities increase appropriately in response to mild reductions in vessels' luminal diameter but then the vessels begin to accommodate less responsively as the stenosis progresses. Flow velocities and changes thereof among intracranial stenosis patients may indicate acute hemodynamically significant changes such as those incurred from infarcts by artery-to-artery embolism $[33,44,45]$. Serial TCDs can also distinguish between recanalized embolic processes and stenoses [46].

Embolic signals have been detected in vivo in various procedures and in conditions associated with stroke; [47, 48] TCD can detect ES suggestive of an embolic pattern of cortical or multiple infarcts in $33 \%$ of patients with symptomatic middle cerebral artery stenosis [49]. Furthermore, diffusion-weighted sequences in MRI studies have confirmed that abnormal ES patterns on TCD are indicative of an embolic process $[32,50,51]$ while animal models have also supported ES as thrombotic particulate material from artery-to-artery embolism [45]. Though the majority of microemboli is washed out into the venous circulation [52], presence of ES on TCD may indicate active embolization [53, 54]. In extracranial carotid stenosis, ES are more common in symptomatic than asymptomatic patients, correlate with the degree of stenosis, and are associated with a greater risk of recurrent stroke [48]. In intracranial stenosis, most studies have focused on ES from the middle cerebral artery as it is the most accessible for monitoring. Embolic signals are noted in about $30 \%$ of stroke patients in the acute phase [32, 46, 47, 50, 51, 55]. In addition, the presence of ES appears to be prognostic of future events (OR 8.4, $P=0.01$ ) with the majority of recurrent events occurring in the first month [50].

Another emerging modality, HR-MRI, creates sufficiently thin cuts through intracranial vessels that the plaque morphology and stability can be detected [27, 28, 56]. By visualizing enhancing or heterogeneous plaques (akin to extracranial carotid plaque evaluation), one can determine whether they are vulnerable (unstable) or stable plaques based on high-grade or low-grade wall shear forces. Preliminary studies have shown the utility of this modality in assessing plaque characteristics even in anatomically more elusive regions such as the cavernous ICA [57].

Impaired cerebral hemodynamics is a well-established predictor of future strokes among patients with large artery stroke [34-36, 58]. Cerebral perfusion pressure distal to a high-grade stenosis or occlusion may be reduced depending on the adequacy of collateral sources of blood flow. As a result, unless collateral flow is adequate, the delivery of oxygen to the tissue will be impaired. Normally, perfusion pressure is maintained by independent compensatory mechanisms such autoregulatory vasodilation and increased oxygen extraction [59]. Once these mechanisms are exhausted, and cerebral perfusion pressure decreases beyond the limits of compensation, $\mathrm{CBF}$ falls and tissue infarction ensues $[60,61]$. In a small study, photon emission tomography (PET) study, there was strong correlation between MCA occlusion and hemodynamic compromise resulting in reduced oxygen extraction [62].

In addition to anterograde flow, perfusion distal to an arterial stenosis is determined compensatory collateral flow; thus, evaluating collateral circulation is critical to the understanding of stroke risk due to intracranial atherosclerotic disease. Primary collateral pathways provide a route for cerebral blood flow to ischemic regions through existing anastomoses at the circle of Willis; flow through the posterior and anterior communicating artery account for the majority of collateral flow in internal carotid and basilar artery stenosis. These can be visualized and quantified using phase-contrast MRA [38, 63-66]. Collaterals via pial and leptomeningeal (secondary collateral pathways) anastomoses may play an important role in middle cerebral artery stenosis $[34,62]$, which is distal to the circle of Willis (primary) collaterals, and can be assessed using tests of vasomotor reactivity (VMR).

Autoregulatory vasodilation in response to a vasodilatory challenge, such as carbon dioxide $\left(\mathrm{CO}_{2}\right)$, defines VMR [36]. Impaired VMR can be used to identify those patients who are at a high-risk for stroke in both intra- and extracranial large vessel disease [58,67-72]. While the hemodynamics of extracranial large vessel disease has been more extensively investigated [36], hemodynamic compromise associated with intracranial occlusive disease may have even greater significance on outcome. In contrast to extracranial highgrade stenosis, collateral flow heavily depends on leptomeningeal arteries since large collaterals from the circle of Willis may not be recruited with more distal intracranial stenosis. A leptomeningeal collateral flow pattern has been associated with a higher prevalence of impaired VMR [73$75]$. 
Sequential testing also suggests that impaired VMR due to intracranial disease is unlikely to recover on follow-up examinations [36]. In a study evaluating 70 cases with intracranial large vessel occlusion using Xenon-SPECT with acetazolamide challenge, one-third had impaired VMR. The annual risk of ipsilateral stroke with impaired VMR was $17.4 \%$ versus $1.2 \%$ with normal reactivity [68]. TCD with $\mathrm{CO}_{2}$ challenge, performed by measuring the blood flow with breath-holding, evaluates the degree of dilatation that occurs in distal arterioles in response to hypercarbia [73, 74]. Noting the change in MFV in response to $\mathrm{CO}_{2}$ challenge, one can also gauge VMR $[68,73,74,76]$.

Perfusion studies (CT and MRI) using the bolus-track method [77] can measure resting tissue perfusion as well as cerebrovascular reserve in patients with intracranial stenosis in response to a challenge [76]. While the optimal parameter to define reversible hypoperfusion (ischemia without infarction) has yet to be validated, mean transit time (MTT) and cerebral blood volume (CBV) have been the most studied and the most consistent. A increase in MTT with preservation of $\mathrm{CBV}$ indicates tissue-at-risk of infarction and may be a marker of high stroke risk [78]. Xenon-CT is also a reliable predictor of cerebrovascular reserve capacity and MTT [79]. PET scans can also assess oxygen extraction fraction within the tissue supplied by the stenotic vessel and provide a marker of advanced hemodynamic failure [67].

By studying cerebral perfusion and cerebrovascular reserve capacity before and after administering the vasoactive agents, one can evaluate the physiologic sequelae of the flow-limiting stenosis as a function of degree of hemodyanamic failure or compromise [80]. Much like diagnostic imaging modalities can complement one other, the available tools to identify physiologic markers are best utilized in combination to inform and calibrate stroke risk. In clinical practice, suspected patients with intracranial stenosis are evaluated with MRA, TCD, and/or CTA with DSA serving a final confirmatory test. While imaging techniques assessing key physiologic parameters associated with intracranial stenosis may prove more important than just an anatomic diagnosis, these techniques require further prospective study [36].

\section{CONCLUSION}

Modern neuroimaging has been critical to improving the capacity to diagnose intracranial stenosis routinely and also holds promise in unraveling putative mechanisms of stroke in this high-risk population. Understanding the strengths and limitations of each imaging tool and its diagnostic utility are essential to accurate and timely diagnosis and can assist in stroke risk stratification. A comprehensive approach that integrates both clinical and radiographic markers of risk will ultimately best.

\section{REFERENCES}

[1] Ducros A, Bousser MG. Reversible cerebral vasoconstriction syndrome. Pract Neurol 2009; 9(5): 256-67.

[2] Herregods N, Beckers R, Van Rattinghe R, Verstraete K. Fibromuscular dysplasia of the carotid artery. JBR-BTR 2008; 91(5): 195-7.

[3] Famakin BM, Chimowitz MI, Lynn MJ, Stern BJ, George MG. WASID Trial Investigators. Causes and severity of ischemic stroke in patients with symptomatic intracranial arterial stenosis. Stroke 2009; 40(6): 1999-2003.
[4] Taqui A, Kamal AK. Strokes in Asians. Pak J Neurol Sci 2007; 2(1): 14-7.

[5] Wong KS, Huang YN, Gao S, Lam WW, Chan YL, Kay R. Intracranial stenosis in Chinese patients with acute stroke. Neurology 1998; 50(3): 812-3.

[6] Prognosis of patients with symptomatic vertebral or basilar artery stenosis. The Warfarin-Aspirin Symptomatic Intracranial Disease (WASID) Study Group. Stroke 1998; 29(7): 1389-92.

[7] Thijs VN, Albers GW. Symptomatic intracranial atherosclerosis: outcome of patients who fail antithrombotic therapy. Neurology 2000; 55(4): 490-7.

[8] Qureshi AI, Ziai WC, Yahia AM, et al. Stroke-free survival and its determinants in patients with symptomatic vertebrobasilar stenosis: a multicenter study. Neurosurgery 2003; 52(5): 1033-9; Discussion $1039-40$

[9] Williams JE, Chimowitz MI, Cotsonis GA, Lynn MJ, Waddy SP WASID Investigators. Gender differences in outcomes among patients with symptomatic intracranial arterial stenosis. Stroke 2007; 38(7): 2055-62.

[10] Waddy SP, Cotsonis G, Lynn MJ, et al. Racial differences in vascular risk factors and outcomes of patients with intracranial atherosclerotic arterial stenosis. Stroke 2009; 40(3): 719-25.

[11] Kim BJ, Lee SH, Kang BS, Yoon BW, Roh JK. Diabetes increases large artery diseases, but not small artery diseases in the brain. $\mathrm{J}$ Neurol 2008; 255(8): 1176-81.

[12] Solberg LA, McGarry PA. Cerebral atherosclerosis in Negroes and Caucasians. Atherosclerosis 1972; 16(2): 141-54.

[13] Nahab F, Cotsonis G, Lynn M, et al. WASID Study Group. Prevalence and prognosis of coexistent asymptomatic intracranial stenosis. Stroke 2008; 39(3): 1039-41.

[14] Kern R, Steinke W, Daffertshofer M, Prager R, Hennerici M. Stroke recurrences in patients with symptomatic $v s$ asymptomatic middle cerebral artery disease. Neurology 2005; 65(6): 859-64.

[15] Bash S, Villablanca JP, Jahan R, et al. Intracranial vascular stenosis and occlusive disease: evaluation with $\mathrm{CT}$ angiography, MR angiography, and digital subtraction angiography. AJNR Am J Neuroradiol 2005; 26(5): 1012-21

[16] Cloft HJ, Joseph GJ, Dion JE. Risk of cerebral angiography in patients with subarachnoid hemorrhage, cerebral aneurysm, and arteriovenous malformation: a meta-analysis. Stroke 1999; 30(2): 317-20.

[17] Martinelli O, Benedetti-Valentini F. Trancranial Doppler: value in clinical practice. Int Angiol 2009; 28(4): 249-53.

[18] Tsivgoulis G, Alexandrov AV, Sloan MA. Advances in transcranial Doppler ultrasonography. Curr Neurol Neurosci Rep 2009; 9(1): 46-54.

[19] Feldmann E, Wilterdink JL, Kosinski A, et al. The Stroke Outcomes and Neuroimaging of Intracranial Atherosclerosis (SONIA) trial. Neurology 2007; 68(24): 2099-106.

[20] Kelley RE, Namon RA, Mantelle LL, Chang JY. Sensitivity and specificity of transcranial Doppler ultrasonography in the detection of high-grade carotid stenosis. Neurology 1993; 43(6): 1187-91.

[21] Cochran ST. Anaphylactoid reactions to radiocontrast media. Curr Allergy Asthma Rep 2005; 5(1): 28-31.

[22] Mühlenbruch G, Krings T. Comparison of dual-source CT angiography and MR angiography in preoperative evaluation of intra- and extracranial vessels: a pilot study. Eur Radiol 2010 [Epub ahead of print].

[23] Wada K, Kimura K, Minematsu K, Uchino M, Yamaguchi T. Spotty cortical enhancement detected by magnetic resonance imaging: a sign of embolic transient ischemic attack and stroke? J Stroke Cerebrovasc Dis 2001; 10(1): 19-22.

[24] Amin-Hanjani S, Du X, Zhao M, Walsh K, Malisch TW, Charbel FT. Use of quantitative magnetic resonance angiography to stratify stroke risk in symptomatic vertebrobasilar disease. Stroke 2005; 36(6): 1140-5.

[25] Zhao M, Amin-Hanjani S, Ruland S, Curcio AP, Ostergren L, Charbel FT. Regional cerebral blood flow using quantitative MR angiography. AJNR Am J Neuroradiol 2007; 28(8): 1470-3.

[26] Prabhakaran S, Warrior L, Wells KR, Jhaveri MD, Chen M, Lopes DK. The utility of quantitative magnetic resonance angiography in the assessment of intracranial in-stent stenosis. Stroke 2009; 40(3): 991-3.

[27] Turan TN, Bonilha L, Morgan PS, Adams RJ, Chimowitz MI. Intraplaque hemorrhage in symptomatic intracranial atherosclerotic disease. J Neuroimaging. 2009; Nov 11 [Epub ahead of print]. 
[28] Gao H, Long Q, Graves M, Gillard JH, Li ZY. Carotid arterial plaque stress analysis using fluid-structure interactive simulation based on in-vivo magnetic resonance images of four patients. J Biomech 2009; 42(10): 1416-23.

[29] Katz DA, Marks MP, Napel SA, Bracci PM, Roberts SL. Circle of Willis: evaluation with spiral CT angiography, MR angiography, and conventional angiography. Radiology 1995; 195(2): 445-9.

[30] Nguyen-Huynh MN, Wintermark M, English J, et al. How accurate is CT angiography in evaluating intracranial atherosclerotic disease? Stroke 2008; 39(4): 1184-8.

[31] Caplan LR, Hennerici M. Impaired clearance of emboli (washout) is an important link between hypoperfusion, embolism, and ischemic stroke. Arch Neurol 1998; 55(11): 1475-82.

[32] Wong KS, Gao S, Chan YL, et al. Mechanisms of acute cerebral infarctions in patients with middle cerebral artery stenosis: a diffusion-weighted imaging and microemboli monitoring study. Ann Neurol 2002; 52(1): 74-81

[33] Wong KS, Li H, Lam WW, Chan YL, Kay R. Progression of middle cerebral artery occlusive disease and its relationship with further vascular events after stroke. Stroke 2002; 33(2): 532-6.

[34] Derdeyn CP, Powers WJ, Grubb RL Jr. Hemodynamic effects of middle cerebral artery stenosis and occlusion. AJNR Am J Neuroradiol 1998; 19(8): 1463-9.

[35] Yamauchi H, Fukuyama H, Nagahama Y, Nabatame H, Shio H. [Cerebral hemodynamics and risk for recurrent stroke in symptomatic internal carotid artery occlusion]. Rinsho Shinkeigaku 1999; 39(5): 513-9.

[36] Derdeyn CP, Grubb RL Jr, Powers WJ. Cerebral hemodynamic impairment: methods of measurement and association with stroke risk. Neurology 1999; 53(2): 251-9.

[37] Zhao M, Charbel FT, Alperin N, Loth F, Clark ME. Improved phase-contrast flow quantification by three-dimensional vessel localization. Magn Reson Imaging 2000; 18(6): 697-706.

[38] Vanninen RL, Manninen HI, Partanen PL, Vainio PA, Soimakallio S. Carotid artery stenosis: clinical efficacy of MR phase-contrast flow quantification as an adjunct to MR angiography. Radiology 1995; 194(2): 459-67.

[39] Charbel FT, Guppy KH, Zhao M, Clark ME. Computerized hemodynamic evaluation of the cerebral circulation for bypass. Neurosurg Clin N Am 2001; 12(3): 499-508, viii.

[40] Hendrikse J, Rutgers DR, Klijn CJ, Eikelboom BC, van der Grond J. Effect of carotid endarterectomy on primary collateral blood flow in patients with severe carotid artery lesions. Stroke 2003; 34(7): $1650-4$.

[41] Langer DJ, Lefton DR, Ostergren L, et al. Hemispheric revascularization in the setting of carotid occlusion and subclavian steal: a diagnostic and management role for quantitative magnetic resonance angiography? Neurosurgery 2006; 58(3): 528-33; Discussion 528-33.

[42] Guppy KH, Charbel FT, Corsten LA, Zhao M, Debrun G. Hemodynamic evaluation of basilar and vertebral artery angioplasty. Neurosurgery 2002; 51(2): 327-33; Discussion 333-4.

[43] Brisman JL. Wingspan stenting of symptomatic extracranial vertebral artery stenosis and perioperative evaluation using quantitative magnetic resonance angiography: report of two cases. Neurosurg Focus 2008; 24(2): E14.

[44] Forteza AM, Koch S, Romano JG, Babikian VL. Detection of microembolus with transcranial doppler. Rev Neurol 2000; 31(11): 1046-53.

[45] Russell D, Madden KP, Clark WM, Sandset PM, Zivin JA. Detection of arterial emboli using Doppler ultrasound in rabbits. Stroke 1991; 22(2): 253-8.

[46] Diehl RR, Samii C, Diehl A. Dynamics and embolic activity of symptomatic intra-cranial cerebral artery stenoses. Acta Neurol Scand 2002; 106(3): 173-81.

[47] Dittrich R, Ritter MA, Kaps M, et al. The use of embolic signal detection in multicenter trials to evaluate antiplatelet efficacy: signal analysis and quality control mechanisms in the CARESS (Clopidogrel and Aspirin for Reduction of Emboli in Symptomatic carotid Stenosis) trial. Stroke 2006; 37(4): 1065-9.

[48] Markus HS, Ackerstaff R, Babikian V, et al. Intercenter agreement in reading Doppler embolic signals. A multicenter international study. Stroke 1997; 28(7): 1307-10.

[49] Segura T, Serena J, Castellanos M, Teruel J, Vilar C, Dávalos A. Embolism in acute middle cerebral artery stenosis. Neurology 2001; 56(4): 497-501.
[50] Gao S, Wong KS, Hansberg T, Lam WW, Droste DW, Ringelstein EB. Microembolic signal predicts recurrent cerebral ischemic events in acute stroke patients with middle cerebral artery stenosis. Stroke 2004; 35(12): 2832-6.

[51] Gao S, Lam WW, Chan YL, Liu JY, Wong KS. Optimal values of flow velocity on transcranial doppler in grading middle cerebral artery stenosis in comparison with magnetic resonance angiography. J Neuroimaging 2002; 12: 213-8.

[52] Russell GB, Graybeal JM. Detection of venous air embolism: comparison of oxygenation and ventilation monitoring methods in dogs. J Neurosurg Anesthesiol 1992; 4(1): 36-40.

[53] Markus HS, Droste DW, Brown MM. Detection of asymptomatic cerebral embolic signals with Doppler ultrasound. Lancet 1994; 343(8904): 1011-2.

[54] Georgiadis D, Wenzel A, Lehmann D, et al. Influence of oxygen ventilation on Doppler microemboli signals in patients with artificial heart valves. Stroke 1997; 28(11): 2189-94.

[55] Droste DW, Lakemeier S, Wichter T, et al. Optimizing the technique of contrast transcranial Doppler ultrasound in the detection of right-to-left shunts. Stroke 2002; 33(9): 2211-6.

[56] Granada JF, Kaluza GL, Wilensky RL, Biedermann BC, Schwartz RS, Falk E. Porcine models of coronary atherosclerosis and vulnerable plaque for imaging and interventional research. EuroIntervention 2009; 5(1): 140-8.

[57] Hiu T, Hayashi K, Kitagawa N, et al. Intracranial internal carotid artery stenosis with vulnerable plaques successfully treated by stenting under cerebral protection. Clin Neurol Neurosurg 2008; 110(10): 1031-4

[58] Silvestrini M, Vernieri F, Pasqualetti P, et al. Impaired cerebral vasoreactivity and risk of stroke in patients with asymptomatic carotid artery stenosis. JAMA 2000; 283(16): 2122-7.

[59] Powers WJ, Grubb RL Jr, Raichle ME. Clinical results of extracranial-intracranial bypass surgery in patients with hemodynamic cerebrovascular disease. J Neurosurg 1989; 70(1): 61-7.

[60] Altman DI, Lich LL, Powers WJ. Brief inhalation method to measure cerebral oxygen extraction fraction with PET: accuracy determination under pathologic conditions. J Nucl Med 1991; 32(9): 1738-41.

[61] Derdeyn CP, Videen TO, Yundt KD, et al. Variability of cerebral blood volume and oxygen extraction: stages of cerebra haemodynamic impairment revisited. Brain 2002; 125(Pt 3): 595 607.

[62] Liebeskind DS. Collateral circulation. Stroke 2003; 34(9): 2279-84.

[63] Van der Grond J, Eikelboom BC, Mali WP. Flow-related anaerobic metabolic changes in patients with severe stenosis of the internal carotid artery. Stroke 1996; 27(11): 2026-32.

[64] Van Everdingen KJ, Klijn CJ, Kappelle LJ, Mali WP, van der Grond J. MRA flow quantification in patients with a symptomatic internal carotid artery occlusion. The Dutch EC-IC Bypass Study Group. Stroke 1997; 28(8): 1595-600.

[65] Hartkamp MJ, van Der Grond J, van Everdingen KJ, Hillen B, Mali WP. Circle of Willis collateral flow investigated by magnetic resonance angiography. Stroke 1999; 30(12): 2671-8.

[66] Zwanenburg JJ, Hendrikse J, Takahara T, Visser F, Luijten PR. MR angiography of the cerebral perforating arteries with magnetization prepared anatomical reference at $7 \mathrm{~T}$ : comparison with time-of-flight. J Magn Reson Imaging 2008; 28(6): 1519-26.

[67] Sánchez-Arjona MB, Sanz-Fernández G, Franco-Macías E, GilPeralta A. Cerebral hemodynamic changes after carotid angioplasty and stenting. AJNR Am J Neuroradiol 2007; 28(4): 640-4.

[68] Uzunca I, Asil T, Balci K, Utku U. Evaluation of vasomotor reactivity by transcranial Doppler sonography in patients with acute stroke who have symptomatic intracranial and extracranial stenosis. J Ultrasound Med 2007; 26(2): 179-85.

[69] Terada S, Ishizu H, Tanabe Y, et al. Plaque-like structures and arteriosclerotic changes in "diffuse neurofibrillary tangles with calcification" (DNTC). Acta Neuropathol 2001; 102(6): 597-603.

[70] Cullinane M, Markus HS. Evaluation of a $1 \mathrm{MHz}$ transducer for transcranial Doppler ultrasound including embolic signal detection. Ultrasound Med Biol 2001; 27(6): 795-800.

[71] Pyner S, Coney A, Marshall JM. The role of free radicals in the muscle vasodilatation of systemic hypoxia in the rat. Exp Physiol 2003; 88(6): 733-40. 
[72] Reinhard M, Waldkircher Z, Timmer J, Weiller C, Hetzel A. Cerebellar autoregulation dynamics in humans. J Cereb Blood Flow Metab 2008; 28(9): 1605-12.

[73] Ringelstein EB, Weiller C, Weckesser M, Weckesser S. Cerebral vasomotor reactivity is significantly reduced in low-flow as compared to thromboembolic infarctions: the key role of the circle of Willis. J Neurol Sci 1994; 121(1): 103-9.

[74] Ozgur HT, Kent Walsh T, Masaryk A, et al. Correlation of cerebrovascular reserve as measured by acetazolamide-challenged SPECT with angiographic flow patterns and intra- or extracranial arterial stenosis. AJNR Am J Neuroradiol 2001; 22(5): 928-36.

[75] Vernieri F, Pasqualetti P, Matteis M, et al. Effect of collateral blood flow and cerebral vasomotor reactivity on the outcome of carotid artery occlusion. Stroke 2001; 32(7): 1552-8.

[76] Kim E, Sohn CH, Na DG, et al. Perfusion computed tomography evaluation of cerebral hemodynamic impairment in patients with unilateral chronic steno-occlusive disease: a comparison with the acetazolamide challenge 99mTc-hexamethylpropyleneamine oxime single-photon emission computed tomography. J Comput Assist Tomogr 2009; 33(4): 546-51.

[77] Doege CA, Tavakolian R, Kerskens CM, et al. Perfusion and diffusion magnetic resonance imaging in human cerebral venous thrombosis. J Neurol 2001; 248(7): 564-71.

[78] Sims JR, Gharai LR, Schaefer PW, et al. ABC/2 for rapid clinical estimate of infarct, perfusion, and mismatch volumes. Neurology 2009; 72(24): 2104-10.

[79] Schubert GA, Weinmann C, Seiz M, et al. Cerebrovascular insufficiency as the criterion for revascularization procedures in selected patients: a correlation study of xenon contrast-enhanced CT and PWI. Neurosurg Rev 2009; 32(1): 29-35; discussion 35-6.

[80] O JH, Jang KS, Yoo IeR, et al. Assessment of cerebrovascular reserve before and after STA-MCA bypass surgery by SPECT and SPM analysis. Korean J Radiol 2007; 8(6): 458-65.

(C) Joshi and Prabhakaran; Licensee Bentham Open.

This is an open access article licensed under the terms of the Creative Commons Attribution Non-Commercial License (http://creativecommons.org/licenses/by$\mathrm{nc} / 3.0 /$ ) which permits unrestricted, non-commercial use, distribution and reproduction in any medium, provided the work is properly cited. 